\title{
An empirical continuous positive airway pressure trial for suspected obstructive sleep apnea
}

\author{
Robert P Skomro MD FRCPC ${ }^{1}$, David J Cotton MD FRCPC ${ }^{1}$, John A Gjevre MD FRCPC ${ }^{1}$, Vaneeta K Grover MA MSc ${ }^{2}$, \\ Brian D MCNab MD FRCPC ${ }^{1}$, John K Reid MD FRCPC ${ }^{1}$, Heather A Ward MD FRCPC ${ }^{3}$
}

\begin{abstract}
RP Skomro, DJ Cotton, JA Gjevre, et al. An empirical continuous positive airway pressure trial for suspected obstructive sleep apnea. Can Respir J 2007;14(3):159-163.
\end{abstract}

BACKGROUND: Standard practice in obstructive sleep apnea (OSA) management requires that a positive diagnostic, overnight polysomnography (PSG) test be obtained before initiating treatment. However, long waiting times due to lack of access to PSG testing facilities may delay the initiation of definitive treatment for OSA.

OBJECTIVES: To evaluate the response of patients who had a high clinical suspicion for OSA and who were waiting for a PSG test to an empirical continuous positive airway pressure (CPAP) trial.

METHODS: A retrospective study of all patients who had been offered empirical CPAP therapy for suspected OSA was conducted. After outpatient assessment, 183 patients with a high pretest probability of having OSA began empirical CPAP testing using an arbitrary CPAP pressure. The presence of OSA, the accuracy of empirical CPAP pressure prescription, the adherence to empirical CPAP and the improvement in daytime somnolence were evaluated at the time of PSG.

RESULTS: Of 183 patients on a CPAP trial, 91\% had OSA, which was at least moderate (more than 15 apneas and hypopneas per hour of sleep) in $75 \%$ of the patients. Eighty per cent of the patients had significant daytime somnolence (Epworth Sleepiness Scale [ESS] greater than 10, mean \pm SD ESS 14 \pm 5 ), which improved with CPAP (ESS $9.0 \pm 5, \mathrm{P}<0.01$ ). In $40 \%$ of the patients, the arbitrary CPAP pressure was lower than that determined by manual titration. Adherence to a trial of CPAP (longer than $2 \mathrm{~h} /$ night) predicted OSA with a sensitivity of $82 \%$ and a specificity of $41 \%$; the positive and negative predictive values were $92 \%$ and $22 \%$, respectively.

CONCLUSIONS: At the time of PSG testing, OSA was present in $91 \%$ of the patients who had received empirical CPAP. An empirical CPAP provided satisfactory interim treatment for excessive somnolence, despite the fact that the CPAP pressure was suboptimal in $40 \%$ of the patients.

Key Words: Continuous positive airway pressure; CPAP responsiveness; CPAP trial; Empirical CPAP; Obstructive sleep apnea

In obstructive sleep apnea (OSA), the intermittent, repetitive obstructions of the oropharynx during sleep decrease arterial oxygen saturation, increase sympathetic discharge and cause sleep disruption (1). Up to $20 \%$ of adults have at least mild OSA, most of whom have not yet been diagnosed $(2,3)$. There are potentially serious adverse consequences of OSA, including a greater risk of hypertension, cardiovascular disease, the metabolic syndrome and traffic or work-related accidents (4-7). The first line of treatment for OSA is continuous

\section{Un essai empirique de pression expiratoire positive continue en cas de présomption d'apnée obstructive du sommeil}

\begin{abstract}
HISTORIQUE : Selon la pratique standard dans le traitement de l'apnée obstructive du sommeil (AOS), il faut obtenir un diagnostic positif au moyen d'une polysomnographie de nuit (PS) avant d'entreprendre le traitement. Cependant, les temps d'attente prolongés causés par l'absence d'accès aux installations de PS peuvent retarder le début du traitement officiel de l'AOS.

OBJECTIFS : Évaluer la réponse des patients dont la présomption clinique d'AOS était élevée et qui attendaient de subir une PS à un essai empirique de pression expiratoire positive continue (PEPC).

MÉTHODOLOGIE : On a mené une étude rétrospective de tous les patients à qui on avait offert un traitement empirique de PEPC en raison d'une présomption d'AOS. Après l'évaluation en consultations externes, 183 patients présentant une probabilité élevée d'AOS ont entrepris l'essai de PEPC empirique au moyen d'une unité de PEPC arbitraire. On a évalué la présence d'AOS, l'exactitude de la prescription d'une unité de PEPC empirique, le respect de la PEPC empirique et l'amélioration de la somnolence pendant le jour au moment de la PS.

RÉSULTATS : Des 183 patients subissant l'essai de PEPC, 91 \% étaient atteints d'AOS, dont $75 \%$ des patients atteints d'une AOS au moins modérée (plus de 15 apnées et hypopnées par heure de sommeil). Quatrevingts pour cent des patients présentaient une somnolence importante pendant le jour (échelle de somnolence d'Epworth [ÉSE] supérieure à 10, moyenne \pm ÉT $14 \pm 5$ ), qui s'atténuait avec la PEPC (ÉSE 9,0 $\pm 5, \mathrm{P}<0,01$ ). Chez $40 \%$ des patients, l'unité de PEPC arbitraire était inférieure à celle mesurée par titrage manuel. Le respect d'un essai de PEPC (plus de deux heures par nuit) prédisait l'AOS avec une sensibilité de $82 \%$ et une spécificité de $41 \%$. Les valeurs prédictives positives et négatives étaient de $92 \%$ et $22 \%$, respectivement.

CONCLUSIONS : Au moment de l'essai de PS, on constatait une AOS chez $91 \%$ des patients qui avaient reçu une PEPC empirique. Une PEPC empirique assurait un traitement provisoire satisfaisant de la somnolence excessive, même si la PEPC était sous-optimale chez 40 \% des patients.
\end{abstract}

positive airway pressure (CPAP), which improves daytime alertness and quality of life, and reduces both the rate of traffic crashes and overall medical costs (7-9).

Current practice guidelines recommend overnight inlaboratory polysomnography (PSG) to diagnose OSA before beginning CPAP treatment (10). However, in some jurisdictions, restricted access to PSG testing facilities forces patients to wait months or even years for definitive diagnosis and treatment (11). Given the potentially adverse consequences of

\footnotetext{
${ }^{1}$ Division of Respirology, Critical Care and Sleep Medicine; ${ }^{2}$ Department of Community Health and Epidemiology; ${ }^{3}$ Division of General Internal Medicine, University of Saskatchewan, Saskatoon, Saskatchewan

Correspondence and reprints: Dr Robert P Skomro, Royal University Hospital, Division of Respirology, Critical Care and Sleep Medicine, 103 Hospital Drive, Ellis Hall, Room 563, Saskatoon, Saskatchewan S7N OW8. Telephone 306-966-2475, fax 306-966-8694, e-mail r.skomro@usask.ca
} 
delaying treatment for OSA and the relatively minor adverse effects of CPAP treatment, we resorted to initiating empirical CPAP treatment (after initial outpatient evaluation) using an arbitrary CPAP pressure in selected patients with a high likelihood of having OSA. The outcome of empirical CPAP treatment was retrospectively evaluated at the time of diagnostic PSG testing.

\section{Study group and protocol}

\section{METHODS}

All patients referred for suspected OSA were evaluated in the outpatient setting by 13 physicians (11 respirologists and two neurologists) during a three-year period (2000 to 2003). The global clinical evaluation took into consideration symptoms of OSA (snoring, witnessed apneas and daytime somnolence), body habitus (body mass index [BMI] and neck size), comorbid conditions associated with OSA (hypertension, coronary disease, stroke and diabetes) and physical examination findings. Sixtyfive patients (36\%) underwent an overnight screening oximetry test at home. Patients commenced empirical CPAP if there was high clinical suspicion of OSA, if there was low likelihood of another sleep disorder and if the patient was willing to begin nightly CPAP treatment in the home (after explanation of the risks and benefits). The patient received the CPAP machine at no charge, and purchased the CPAP mask and humidifier (if needed). The prescribing physician chose an arbitrary CPAP pressure (ranging from $7 \mathrm{~cm} \mathrm{H}_{2} \mathrm{O}$ to $12 \mathrm{~cm} \mathrm{H}_{2} \mathrm{O}$ ), taking into account BMI, oropharyngeal crowding and neck size. Exclusion criteria included an in-hospital consultation, the presence of an occupationally sensitive job (eg, commercial drivers and pilots), high suspicion of another primary sleep disorder (eg, narcolepsy and restless legs syndrome), and the presence of respiratory or congestive heart failure. At the time of PSG testing, height and weight were measured and BMI was calculated. The patients completed the Epworth Sleepiness Scale (ESS) before CPAP was begun and at the time of PSG testing (12). CPAP responsiveness was defined as a change in ESS with CPAP treatment of at least five points. CPAP adherence was evaluated by accessing data stored in the CPAP units at the time of the PSG. The present study was approved by the University of Saskatchewan (Saskatoon, Saskatchewan) Biomedical Research Ethics Board.

\section{Equipment}

A supervised, in-laboratory PSG included three electroencephalography leads; two electro-oculography leads; sub-mental electromyography (EMG); pulse oximetry; measurement of airflow (a pressure sensor or thermistor), chest and rib cage movements (piezoelectric belts); notation of snoring (a vibration sensor); diaphragmatic EMG; anterior tibialis EMG; one-lead electrocardiography; and notation of sleep position. Signals were digitally recorded using Sandman diagnostic program (Nellcor Puritan Bennett Inc, Ontario). Standardized sleep staging and scoring for sleep apnea was undertaken by PSG technicians and confirmed by a sleep medicine physician $(13,14)$. OSA was defined as five or more obstructive apneas and hypopneas per hour of sleep (apnea-hypopnea index [AHI]). Obstructive hypopnea was defined as having at least a 50\% decrease in oronasal flow for $10 \mathrm{~s}$ or longer, with the presence of respiratory effort and either a 3\% decrease in oxygen saturation or a significant activation in electroencephalography (14). Severe OSA was defined as having an AHI higher than 30; moderate OSA was defined as an AHI 30 or lower but higher than 15; and mild OSA was defined as an AHI 15 or lower but higher than five.
Patients were asked to refrain from CPAP use in the two nights before PSG testing to reduce a washout effect, which could have reduced the severity of OSA. Patients with at least moderate OSA during the first $4 \mathrm{~h}$ of sleep had 'split-night' PSG (15). If OSA was mild, a full diagnostic study was performed, followed by a second full-night PSG test with CPAP titration. During CPAP titration, the CPAP pressure was adjusted to abolish obstructive apneas, hypopneas and desaturations. The empirical CPAP pressure was considered optimal if it was the same as or higher than the manual CPAP titration but was considered suboptimal if it was lower than the CPAP titration pressure. All patients were interviewed by a sleep medicine physician the morning after the PSG test.

\section{Statistical analysis}

Continuous data were presented as means \pm SDs, and categorical data were presented as frequencies (per cents). Categorical variables were compared using a $\chi^{2}$ analysis. A paired two-tailed $t$ test was used for comparing continuous variables before CPAP with continuous variables after CPAP. A $t$ test for two independent samples was used to compare continuous variables between groups. Correlations were assessed using linear regression analysis.

Statistical significance was defined as $\mathrm{P}<0.05$. Statistical analysis was completed using SPSS version 12.0 (SPSS Inc, USA).

\section{RESULTS}

One hundred eighty-three patients (143 men, mean [ \pm SD] $51 \pm 11$ years, mean BMI $37 \pm 8 \mathrm{~kg} / \mathrm{m}^{2}$ and mean ESS $14 \pm 5$ ), who were prescribed empirical CPAP at the initial outpatient assessment, were evaluated at the time of PSG testing. The mean waiting period for PSG was $246 \pm 258$ days (ranging from less than one month in $5 \%$ of patients to longer than six months in $48 \%$ of patients); mean length of CPAP use was $169 \pm 117$ nights ( $63 \%$ of patients had longer than two months of nightly use).

At the time of PSG testing, OSA was present in 166 of the 183 patients (91\%) receiving empirical CPAP. Of those, 161 patients continued to use CPAP after PSG, and five patients were prescribed bilevel PAP because of hypoventilation or intolerance to high CPAP pressure. The mean AHI was $42 \pm 34$, and OSA was found to be severe in $53 \%$ of patients, moderate in $22 \%$ and mild in $16 \%$. The mean CPAP adherence was $4.6 \pm 2.5 \mathrm{~h} /$ night. No serious adverse reactions were reported. On empirical CPAP, the ESS decreased significantly $(14 \pm 5$ versus $9 \pm 5, \mathrm{P}<0.05)$. Fifty-three per cent of the patients on empirical CPAP were CPAP responsive (defined as a reduction in the ESS by at least five points). Patients who underwent overnight oximetry before CPAP therapy were similar in age, sex, BMI, AHI and CPAP pressures to those who were prescribed CPAP without oximetry.

In patients diagnosed with OSA at the time of PSG testing $(n=166)$ (Table 1), the mean AHI was $46 \pm 33$. There was significant improvement in the ESS with empirical CPAP (mean decrease of $5.0 \pm 0.5$ points, $\mathrm{P}<0.01$ ); $54 \%$ of patients ( 83 of 155 ) were CPAP responsive. The change in the ESS with treatment correlated weakly with AHI $(r=0.23, \mathrm{P}<0.01)$. Mean CPAP adherence was $4.7 \pm 2.5 \mathrm{~h} /$ night. Men and women with OSA who received empirical CPAP were similar in age, ESS and AHI, but women were heavier than men. OSA was more common in men (133 of 143 patients [93\%]) than women (33 of 40 patients [83\%]) $(\mathrm{P}<0.05)$ (Table 1). The manually titrated CPAP pressure in OSA patients correlated weakly with BMI: CPAP pressure $\left(\mathrm{cm} \mathrm{H}_{2} \mathrm{O}\right)=$ $6.2+(\mathrm{BMI} \times 0.11)\left(\mathrm{r}^{2}=0.14, \mathrm{P}<0.001\right)$. 
The arbitrary CPAP pressure in OSA patients was significantly lower than the CPAP pressure determined during PSG testing by manual titration $\left(9.7 \pm 1.4 \mathrm{~cm} \mathrm{H} \mathrm{H}_{2} \mathrm{O}\right.$ versus $\left.10.1 \pm 2.2 \mathrm{~cm} \mathrm{H}_{2} \mathrm{O}, \mathrm{P}<0.05\right)$. The arbitrary CPAP pressure was lower than the manual pressure in $40 \%$ of patients. In those with suboptimal CPAP pressure, the absolute difference in CPAP pressure was frequently small $\left(2 \mathrm{~cm} \mathrm{H}_{2} \mathrm{O}\right.$ or less in $50 \%$ of patients). OSA patients with suboptimal CPAP pressure had a higher AHI than those with optimal CPAP pressure, but there were no significant differences between these groups in age, BMI, decrease in ESS with CPAP treatment or CPAP adherence (Table 1). Despite suboptimal CPAP pressure, improvement in the ESS with CPAP treatment and the CPAP adherence $(4.8 \pm 2.5 \mathrm{~h} / \mathrm{night})$ were similar to those optimally treated with empirical CPAP.

Seventeen patients (10 men and seven women) on empirical CPAP did not have OSA. Among them, five patients who had primary snoring and/or probable upper airway resistance syndrome had used empirical CPAP for at least six months. One patient had periodic breathing and another had alveolar hypoventilation. Both patients were noncompliant with CPAP. In the remaining 10 patients (5.5\%) who did not have sleep-disordered breathing, four (2\%) had periodic leg movements and six (3\%) had a completely normal PSG; five of these patients did not adhere to CPAP.

In the 154 patients whose adherence was documented, 79\% (122 of 154 patients) had adhered to CPAP (at least $2 \mathrm{~h} /$ night of CPAP use). Among those who adhered to empirical CPAP, 92\% (112 of 122 patients) had OSA (true positives) when tested by PSG but $8 \%$ (10 of 122 patients) did not (false positives). Alternatively, in $21 \%$ of the patients who did not adhere to empirical CPAP, $78 \%$ (25 of 32 patients) had OSA (false negatives), which was severe in $68 \%$. Only $22 \%$ of the nonadherent patients (seven of 32) did not have OSA (true negatives). Adherence to a trial of CPAP (greater than $2 \mathrm{~h} /$ night) predicted OSA with a sensitivity of $82 \%$ and a specificity of $41 \%$; the positive and negative predictive values were $92 \%$ and $22 \%$, respectively.

\section{DISCUSSION}

In the present study, $91 \%$ of patients who had been started on empirical CPAP had OSA. Their CPAP adherence and improvement in subjective somnolence were comparable with those prescribed CPAP in the traditional manner after in-laboratory CPAP titration $(16,17)$. The patients enrolled in the CPAP trial waited several months (longer than 180 days in $48 \%$ of patients) for PSG testing. The mean waiting time was longer in a comparable group of patients $(n=119)$ not on empirical CPAP who underwent PSG testing at approximately the same time (mean wait time of $600 \pm 700$ days).

Our study has important limitations. The patients who were selected for empirical CPAP constituted a minority of our outpatients referred for OSA (9\%). In addition, the patients were mostly obese men who had classic findings of loud, habitual snoring and somnolence. There may have been some residual effects of recent CPAP use, which was discontinued two nights before PSG testing. This could have led to underestimation of the AHI (18). Despite this, most patients had at least moderate OSA (AHI higher than 15 in $75 \%$ of patients). Global clinical assessment by physicians with expertise in sleep medicine and patient participation in the decision resulted in only a $9 \%$ false-positive rate for OSA. Our success in recruiting
TABLE 1

Characteristics of patients diagnosed with obstructive sleep apnea

\begin{tabular}{|c|c|c|c|c|c|}
\hline & \multirow[b]{2}{*}{$\begin{array}{c}\text { Total } \\
(n=166)\end{array}$} & \multirow[b]{2}{*}{$\begin{array}{c}\text { Men } \\
(n=133)\end{array}$} & \multirow[b]{2}{*}{$\begin{array}{c}\text { Women } \\
(n=33)\end{array}$} & \multicolumn{2}{|c|}{ Empirical CPAP } \\
\hline & & & & $\begin{array}{c}\text { Optimal } \\
(n=89)\end{array}$ & $\begin{array}{l}\text { Suboptimal } \\
(n=72)\end{array}$ \\
\hline Age (years) & $51 \pm 11$ & $51 \pm 11$ & $50 \pm 10$ & $50 \pm 11$ & $52 \pm 10$ \\
\hline BMI $\left(\mathrm{kg} / \mathrm{m}^{2}\right)$ & $37 \pm 8$ & $36 \pm 6^{*}$ & $44 \pm 11$ & $36 \pm 7$ & $39 \pm 7$ \\
\hline Apnea-hypopnea index & $\times \quad 46 \pm 33$ & $47 \pm 34$ & $40 \pm 30$ & $33 \pm 26^{\dagger}$ & $61 \pm 35$ \\
\hline ESS before CPAP & $14 \pm 5$ & $15 \pm 5$ & $14 \pm 5$ & $15 \pm 5$ & $14 \pm 5$ \\
\hline ESS after CPAP & $9 \pm 5$ & $9 \pm 5$ & $9 \pm 4$ & $9 \pm 4$ & $9 \pm 5$ \\
\hline $\begin{array}{l}\text { Arbitrary CPAP } \\
\text { pressure }\left(\mathrm{cm} \mathrm{H}_{2} \mathrm{O}\right)\end{array}$ & $9.7 \pm 1.4$ & $9.8 \pm 1.3$ & $9.5 \pm 1.5$ & $10.2 \pm 2.2^{\dagger}$ & $9.1 \pm 1.4$ \\
\hline $\begin{array}{l}\text { Titration CPAP } \\
\text { pressure }\left(\mathrm{cm} \mathrm{H}_{2} \mathrm{O}\right)\end{array}$ & $10.2 \pm 2.2$ & $10.2 \pm 2.2$ & $10.2 \pm 2.2$ & $9.0 \pm 1.5^{\dagger}$ & $11.8 \pm 1.8$ \\
\hline $\begin{array}{l}\text { CPAP pressure } \\
\text { difference }\left(\mathrm{cm} \mathrm{H}_{2} \mathrm{O}\right)\end{array}$ & $0.5 \pm 2.4$ & $0.4 \pm 2.4$ & $0.8 \pm 2.4$ & $-1.3 \pm 1.2$ & $2.6 \pm 1.5$ \\
\hline $\begin{array}{l}\text { CPAP adherence } \\
\text { (h/night) }\end{array}$ & $4.7 \pm 2.4$ & $4.6 \pm 2.3$ & $5.3 \pm 3.0$ & $4.7 \pm 2.4$ & $4.8 \pm 2.5$ \\
\hline
\end{tabular}

Values are expressed as mean $\pm S D$. ${ }^{*} P<0.05$ men versus women; ${ }^{\dagger} P<0.05$ optimal versus suboptimal continuous positive airway pressure (CPAP). AHI Apnea-hypopnea index; BMI Body mass index; ESS Epworth Sleepiness Scale

OSA patients for a CPAP trial may be explained by the fact that all patients were initially screened by a family physician who had referred the patient for evaluation of suspected OSA. The prevalence of OSA in our outpatient sleep clinics is currently high $(67 \%)$. Our results may have been affected by the dropout rate (ie, patients prescribed CPAP who did not attend the PSG test). We have estimated the dropout rate to be $17 \%$. The majority of our patients who started CPAP empirically attended the PSG. It is possible, however, that those who did not attend did not have OSA and did not use CPAP. Alternatively, some patients with OSA may have had a dramatic subjective response to CPAP and decided not to undergo PSG testing.

Another limitation of our study is that patient enrolment depended mainly on the sleep physician's overall clinical judgment. We could have used prediction rules to assess the likelihood of OSA, but most do not reliably discriminate OSA patients (19). They also do not consider factors such as the source of data (reliable partner), recent weight gain, a family history of OSA, the presence and severity of comorbid conditions, patient awareness, and the patients' preferences and commitment to a trial of CPAP. In a study by Rodsutti et al (20), the primary factors in a clinical decision rule were sex, age and BMI (secondary factors were snoring and witnessed apneas or gasping). In retrospect, using this rule, most of our patients would have been at high risk because $78 \%$ were male, $84 \%$ (154 of 183 patients) were 40 years of age or older, $33 \%$ (60 of 183 patients) had a BMI of $40 \mathrm{~kg} / \mathrm{m}^{2}$ or greater and $83 \%$ (154 of 183 patients) had a BMI of $30 \mathrm{~kg} / \mathrm{m}^{2}$ or greater. The physical determinants of OSA may also have predictive value for OSA, but their role in the comprehensive assessment of suspected OSA patients remains controversial and their value in women is not certain $(21,22)$.

Other screening techniques for OSA could have been used. Whitelaw et al (23) found that nocturnal oximetry-based home monitoring was as good as diagnostic PSG testing in allowing physicians to predict which suspected OSA patients would have improved quality of life, measured by the Sleep Apnea Quality of Life Index (24) after a four-week trial of 
automatic CPAP. We obtained simple overnight oximetry tracings in approximately one-third of our patients, which revealed oxygen desaturation of $85 \%$ or less in approximately $60 \%$ of the patients; all but one patient who had oxygen desaturation of less than $85 \%$ had OSA, and severe OSA was present in $64 \%$ of the patients. The use of overnight oximetry as a screening test in our study before empirical CPAP prescriptions may have affected the pretest probability of OSA in this group. Both groups, however, were similar in demographics, prevalence and severity of OSA, and CPAP pressure levels, indicating that the use of oximetry did not influence the accuracy of CPAP pressure prescription or the likelihood that the patient had OSA. Other home monitoring equipment for suspected OSA was not used in the present study (25).

In retrospect, what were the potential risks and benefits of implementing treatment in patients without first confirming OSA by PSG? The majority of OSA patients who adhered to empirical therapy received benefit because $68 \%$ of the patients (71 of 105 patients) were CPAP responsive. We defined 'the response to CPAP' as a change in the ESS of five points or more with CPAP treatment. This definition was arbitrary but was likely to be clinically significant. The ESS is commonly used to assess daytime somnolence, and relates to both objective measures of sleep tendency and OSA severity $(26,27)$.

Although CPAP adherence and CPAP responsiveness suggest a benefit with empirical CPAP, we used an arbitrary CPAP pressure between $7 \mathrm{~cm} \mathrm{H}_{2} \mathrm{O}$ to $12 \mathrm{~cm} \mathrm{H}_{2} \mathrm{O}$, which was suboptimal in $40 \%$ of patients and could have led to only partial benefits. However, CPAP responsiveness in patients receiving suboptimal CPAP pressures was comparable to that reported in randomized, controlled trials of severe OSA patients, and was better than that found with the use of sham CPAP for OSA (28-30). Automatic CPAP devices may have better defined optimal CPAP requirements in the home, but these devices were not available to our patients at the time of the study (31). Alternatively, patients could have adjusted their CPAP pressure based on their perceived need, a practice that may be as effective as in-laboratory manual titration (32).

Suboptimal CPAP pressure appeared to be as effective in improving daytime somnolence as optimal CPAP because CPAP responsiveness and CPAP adherence were similar in both suboptimal and optimal groups (Table 1). Because we did not have data on other outcomes, such as quality of life, objective measures of vigilance or residual AHI, these findings should be interpreted with caution. Our results, however, are similar to those of Hukins (33), who found that the clinical response to arbitrary CPAP $\left(8 \mathrm{~cm} \mathrm{H}_{2} \mathrm{O}\right.$ CPAP for a BMI less than $30 \mathrm{~kg} / \mathrm{m}^{2} ; 10 \mathrm{~cm} \mathrm{H}$ O CPAP for a BMI between $30 \mathrm{~kg} / \mathrm{m}^{2}$ and $35 \mathrm{~kg} / \mathrm{m}^{2}$, and $12 \mathrm{~cm} \mathrm{H} O$ CPAP for a BMI greater than $35 \mathrm{~kg} / \mathrm{m}^{2}$ ) was as good as the response to CPAP using PSG titration, despite the fact that a substantial number of patients in the arbitrary group received lower CPAP pressures than needed as determined by CPAP titration.

The algorithms used to predict effective CPAP pressure are based on BMI and neck circumference, but most also include the AHI (34-36). Manual CPAP titration revealed that only $9 \%$ of patients needed less than $8 \mathrm{~cm} \mathrm{H_{2 }} \mathrm{O}$ and only $12.6 \%$ of patients required greater than $12 \mathrm{~cm} \mathrm{H}_{2} \mathrm{O}$. In retrospect, a higher set arbitrary pressure of $12 \mathrm{~cm} \mathrm{H}_{2} \mathrm{O}$ would have been more satisfactory (treating $88 \%$ of patients). In the 21 patients (12.6\%) who required greater than $12 \mathrm{~cm} \mathrm{H}_{2} \mathrm{O}$ by manual
CPAP titration, two-thirds had a BMI greater than $40 \mathrm{~kg} / \mathrm{m}^{2}$. Hukins' algorithm would not have satisfactorily treated these patients (33). The manual CPAP titration pressure in our study did correlate with BMI in patients with OSA (CPAP pressure $\left.\left[\mathrm{cm} \mathrm{H}_{2} \mathrm{O}\right]=6.2+[\mathrm{BMI} \times 0.11]\right)$, but the correlation was weak, particularly in patients with a BMI greater than $40 \mathrm{~kg} / \mathrm{m}^{2}$.

What were the risks of treating patients who did not have OSA? Seventeen of our patients (9\%) did not have OSA, although five had some evidence of mild sleep-disordered breathing, with loud snoring and daytime somnolence (likely upper airway resistance syndrome) (37). Those patients without OSA were subjected to the inconvenience, costs and possible adverse consequences of nightly CPAP.

Adherence to CPAP may have predicted the presence of OSA. The sensitivity of CPAP adherence for OSA was $82 \%$, and the positive predictive value was $92 \%$. However, $21 \%$ of OSA patients did not adhere to empirical CPAP, and $68 \%$ of those had severe OSA (AHI greater than 30). The CPAP trial could have been more clinically useful if nonadherent patients had been identified quickly and PSG had been completed immediately.

Our experience with empirical CPAP is limited, but we suspect that this practice is not limited to our jurisdiction because access to PSG testing is limited in many countries (11). Senn et al (38) found that a positive CPAP (automatic titration) response (longer than $2 \mathrm{~h}$ of nightly use, plus a willingness to continue CPAP use by questionnaire) had positive and negative predictive values for an AHI greater than 10 of $97 \%$ and $78 \%$, respectively, and was accurate in identifying patients who continued on CPAP for at least four months. In our realworld outpatient experience, the positive predictive value of $92 \%$ was similar to that obtained by Senn et al (38), but our negative predictive value was much lower (22\%). Although the purpose of empirical CPAP was not to replace diagnostic PSG, the low negative predictive value of CPAP adherence for OSA would have reduced its potential diagnostic value.

\section{CONCLUSION}

Ninety-one per cent of patients selected for an empirical CPAP trial on the basis of high clinical suspicion of OSA had OSA by PSG testing. Most patients satisfactorily adhered to treatment and noted improvement in daytime somnolence. However, our arbitrary choice of CPAP pressure was imprecise, resulting in supoptimal CPAP pressures in $40 \%$ of patients. Nevertheless, the response to empirical CPAP appeared satisfactory, even when the arbitrary CPAP pressure was suboptimal. Although the experience from this retrospective case series is not definitive, empirical CPAP did appear to provide satisfactory interim treatment in CPAP-adherent patients who had classic symptoms for OSA while awaiting diagnostic PSG testing. However, the potential for benefit in the OSA patients who adhered to empirical CPAP must be balanced by the potential costs and risks of empirical CPAP treatment in the OSA patients who did not tolerate it, in the non-OSA patients who received it and in the OSA patients who adhered to CPAP but were treated with suboptimal CPAP pressures. Early identification of nonadherent CPAP users followed by immediate PSG testing would, in retrospect, have improved the clinical outcome of the trial. In the setting of limited access to diagnostic PSG testing, this strategy deserves further consideration. 
ACKNOWLEDGEMENTS: The authors thank Leanne King, Mark Bosch and Joseph T Mink for assistance with data collection and analysis. The authors also wish to thank the Saskatoon Health Authority and the Lung Association of Saskatchewan for providing research support for this study, as well as the Saskatchewan Aids for Independent Living for providing CPAP units.

\section{REFERENCES}

1. Fogel RB, Malhotra A, White DP. Sleep. 2: Pathophysiology of obstructive sleep apnoea/hypopnoea syndrome. Thorax 2004;59:159-63.

2. Young T, Peppard PE, Gottlieb DJ. Epidemiology of obstructive sleep apnea: A population health perspective. Am J Respir Crit Care Med 2002;165:1217-39.

3. Netzer NC, Hoegel JJ, Loube D, et al. Prevalence of symptoms and risk of sleep apnea in primary care. Chest 2003;124:1406-14.

4. Peppard PE, Young T, Palta M, et al. Prospective study of the association between sleep-disordered breathing and hypertension. N Engl J Med 2000;342:1378-84.

5. Coughlin SR, Mawdsley L, Mugarza JA, Calverley PM, Wilding JP. Obstructive sleep apnoea is independently associated with an increased prevalence of metabolic syndrome. Eur Heart J 2004;25:735-41.

6. Masa JF, Rubio M, Findley LJ. Habitually sleepy drivers have a high frequency of automobile crashes associated with respiratory disorders during sleep. Am J Respir Crit Care Med 2000;162:1407-12.

7. Sassani A, Findley LJ, Kryger M, Goldlust E, George C, Davidson TM. Reducing motor-vehicle collisions, costs, and fatalities by treating obstructive sleep apnea syndrome. Sleep 2004;27:453-8.

8. Gordon P, Sanders MH. Sleep. 7: Positive airway pressure therapy for obstructive sleep apnoea/hypopnoea syndrome. Thorax 2005;60:68-75.

9. Bahammam A, Delaive K, Ronald J, Manfreda J, Roos L, Kryger MH. Health care utilization in males with obstructive sleep apnea syndrome two years after diagnosis and treatment. Sleep 1999;22:740-7.

10. Polysomnography Task Force. American Sleep Disorders Association Standards of Practice Committee. Practice parameters for the indications for polysomnography and related procedures. Sleep 1997;20:406-22.

11. Flemons WW, Douglas NJ, Kuna ST, Rodenstein DO, Wheatley J. Access to diagnosis and treatment of patients with suspected sleep apnea. Am J Respir Crit Care Med 2004;169:668-72.

12. Johns MW. A new method for measuring daytime sleepiness: The Epworth sleepiness scale. Sleep 1991;14:540-5.

13. Rechtschaffen A, Kales A. A manual of standardized terminology, techniques and scoring system for sleep stages of human subjects. Los Angeles: UCLA Brain Information Service/Brain Research Institute, 1968.

14. American Academy of Sleep Medicine Task Force. Sleep-related breathing disorders in adults: Recommendations for syndrome definition and measurement techniques in clinical research. Sleep 1999;22:667-89.

15. Yamashiro Y, Kryger MH. CPAP titration for sleep apnea using a split-night protocol. Chest 1995;107:62-6.

16. Monasterio C, Vidal S, Duran J, et al. Effectiveness of continuous positive airway pressure in mild sleep apnea-hypopnea syndrome. Am J Respir Crit Care Med 2001;164:939-43.

17. Ballester E, Badia JR, Hernandez L, et al. Evidence of the effectiveness of continuous positive airway pressure in the treatment of sleep apnea/hypopnea syndrome. Am J Respir Crit Care Med 1999;159:495-501. (Erratum in 1999;159:1688).
18. Kribbs NB, Pack AI, Kline LR, et al. Effects of one night without nasal CPAP treatment on sleep and sleepiness in patients with obstructive sleep apnea. Am Rev Respir Dis 1993;147:1162-8.

19. Rowley JA, Aboussouan LS, Badr MS. The use of clinical prediction formulas in the evaluation of obstructive sleep apnea. Sleep 2000;23:929-38.

20. Rodsutti J, Hensley M, Thakkinstian A, D'Este C, Attia J. A clinical decision rule to prioritize polysomnography in patients with suspected sleep apnea. Sleep 2004;27:694-9.

21. Tsai WH, Remmers JE, Brant R, Flemons WW, Davies J, Macarthur C. A decision rule for diagnostic testing in obstructive sleep apnea. Am J Respir Crit Care Med 2003;167:1427-32.

22. Lam B, Ip MS, Tench E, Ryan CF. Craniofacial profile in Asian and white subjects with obstructive sleep apnoea. Thorax 2005;60:504-10.

23. Whitelaw WA, Brant RF, Flemons WW. Clinical usefulness of home oximetry compared with polysomnography for assessment of sleep apnea. Am J Respir Crit Care Med 2005;171:188-93

24. Flemons WW, Reimer MA. Development of a disease-specific health-related quality of life questionnaire for sleep apnea. Am J Respir Crit Care Med 1998;158:494-503.

25. Flemons WW, Littner MR, Rowley JA, et al. Home diagnosis of sleep apnea: A systematic review of the literature. An evidence review cosponsored by the American Academy of Sleep Medicine, the American College of Chest Physicians, and the American Thoracic Society. Chest 2003;124:1543-79.

26. Punjabi NM, Bandeen-Roche K, Young T. Predictors of objective sleep tendency in the general population. Sleep 2003;26:678-83.

27. Gottlieb DJ, Whitney CW, Bonekat WH, et al. Relation of sleepiness to respiratory disturbance index: The Sleep Heart Health Study. Am J Respir Crit Care Med 1999;159:502-7.

28. Patel SR, White DP, Malhotra A, Stanchina ML, Ayas NT. Continuous positive airway pressure therapy for treating sleepiness in a diverse population with obstructive sleep apnea: Results of a meta-analysis. Arch Intern Med 2003;163:565-71.

29. Montserrat JM, Ferrer M, Hernandez L, et al. Effectiveness of CPAP treatment in daytime function in sleep apnea syndrome: A randomized controlled study with an optimized placebo. Am J Respir Crit Care Med 2001;164:608-13.

30. Jenkinson C, Davies RJ, Mullins R, Stradling JR. Comparison of therapeutic and subtherapeutic nasal continuous positive airway pressure for obstructive sleep apnoea: A randomised prospective parallel trial. Lancet 1999;353:2100-5.

31. Planes C, D'Ortho MP, Foucher A, et al. Efficacy and cost of home-initiated auto-nCPAP versus conventional nCPAP. Sleep 2003;26:156-60.

32. Fitzpatrick MF, Alloway CE, Wakeford TM, MacLean AW, Munt PW, Day AG. Can patients with obstructive sleep apnea titrate their own continuous positive airway pressure? Am J Respir Crit Care Med 2003;167:716-22.

33. Hukins CA. Arbitrary-pressure continuous positive airway pressure for obstructive sleep apnea syndrome. Am J Respir Crit Care Med 2005; 171:500-5

34. Hoffstein V, Mateika S. Predicting nasal continuous positive airway pressure. Am J Respir Crit Care Med 1994;150:486-8.

35. Oliver Z, Hoffstein V. Predicting effective continuous positive airway pressure. Chest 2000;117:1061-4.

36. Rowley JA, Tarbichi AG, Badr MS. The use of a predicted CPAP equation improves CPAP titration success. Sleep Breath 2005;9:26-32.

37. Guilleminault C, Kim YD, Palombini L, Li K, Powell N. Upper airway resistance syndrome and its treatment. Sleep 2000;23(Suppl 4):S197-200.

38. Senn O, Brack T, Russi EW, Bloch KE. A continuous positive airway pressure trial as a novel approach to the diagnosis of the obstructive sleep apnea syndrome. Chest 2006;129:67-75. 


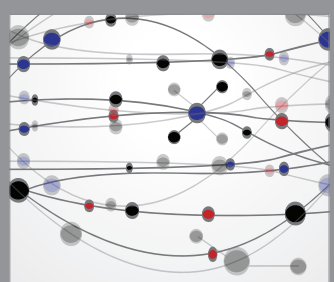

The Scientific World Journal
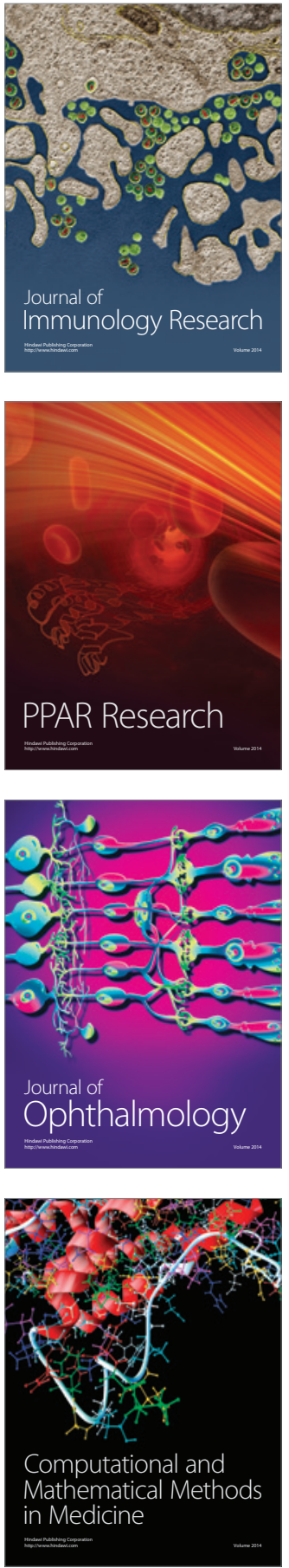

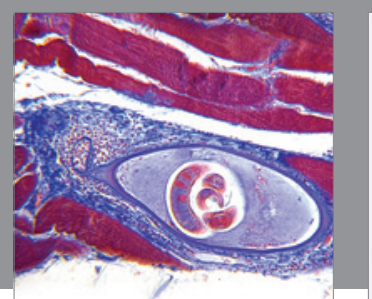

Gastroenterology Research and Practice

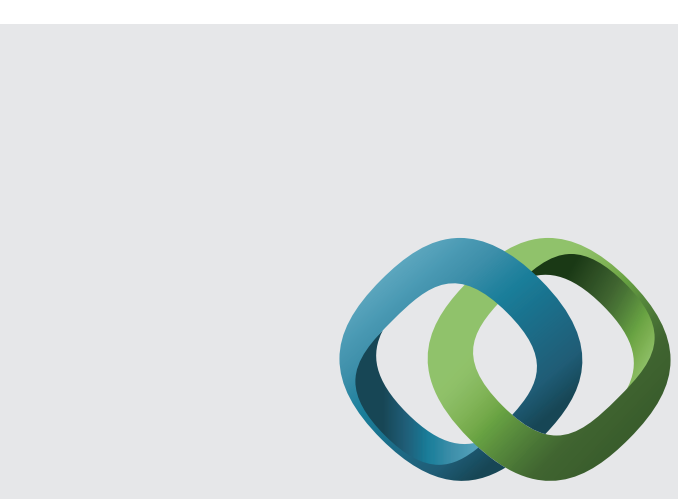

\section{Hindawi}

Submit your manuscripts at

http://www.hindawi.com
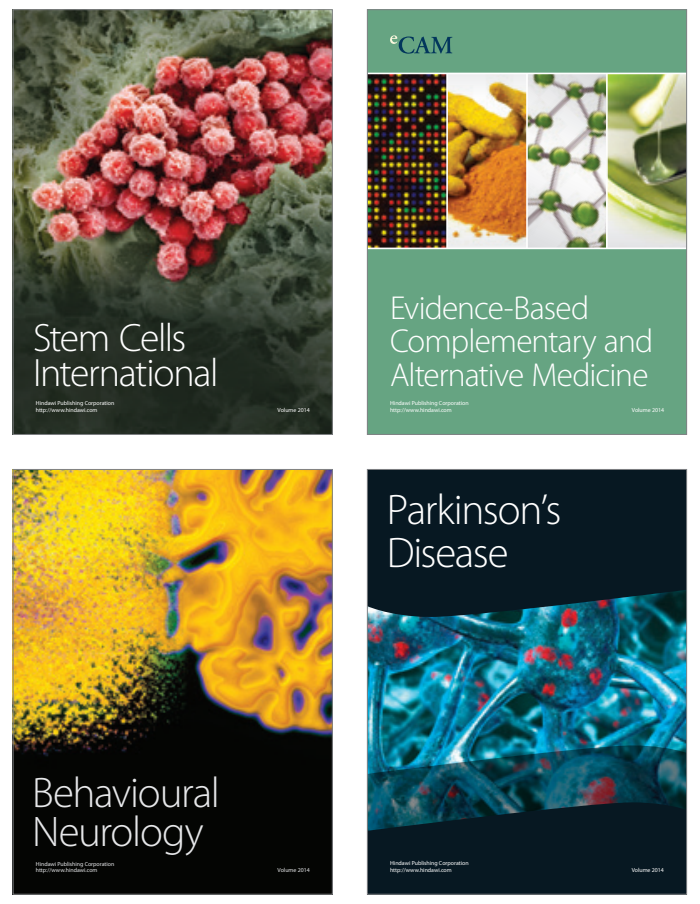


Disease Markers
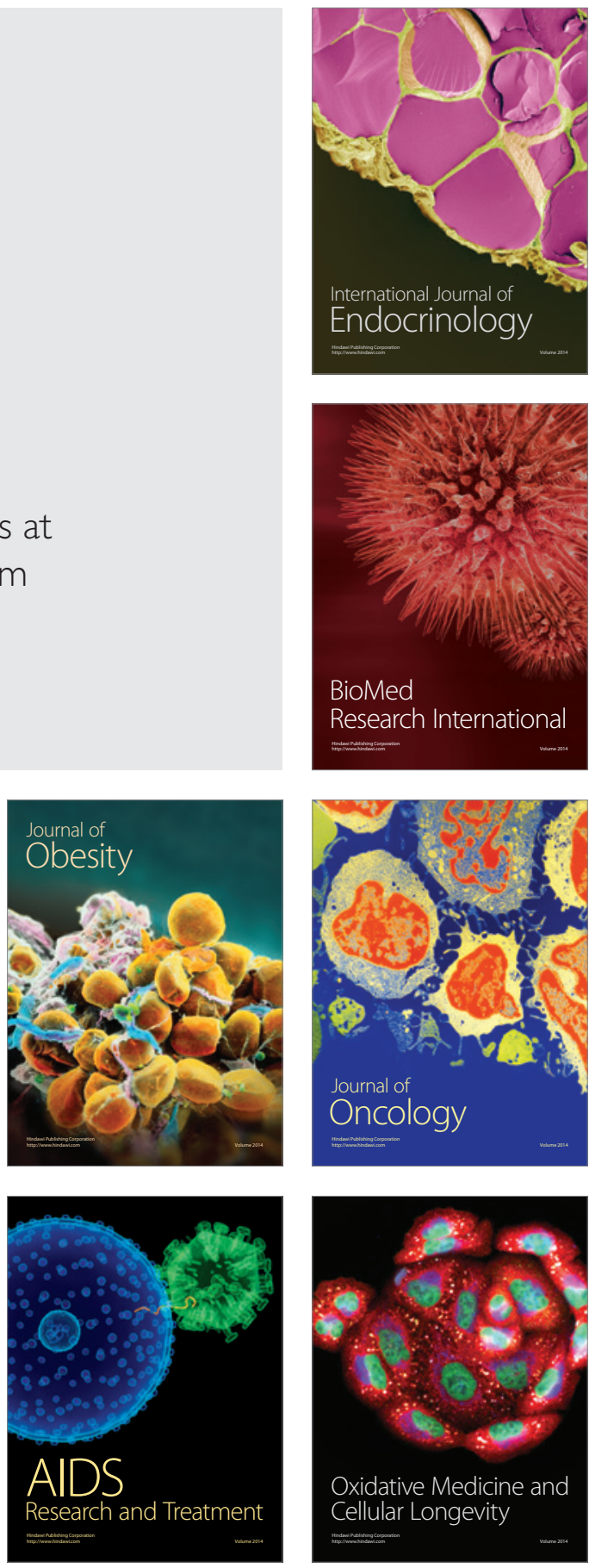